\title{
Health Care Professionals Beliefs and Perceptions on Family-Centered Care in the NICU
}

Vittner $\mathrm{D}^{1,2}$, Parker $\mathrm{M}^{1}$, DeMeo $\mathrm{S}^{1}$, Baxter $\mathrm{A}^{2}$, and McGrath $\mathrm{J}^{3}$

${ }^{1}$ WakeMed Health \& Hospitals, Raleigh, NC USA

${ }^{2}$ University of Connecticut, School of Nursing, Storrs, CT USA

${ }^{3}$ University of Texas, Health Science Center San Antonio, San Antonio, TX USA

\section{Background}

The Neonatal Intensive Care Unit (NICU) is a complex, technology-driven environment where health care professionals have many tasks to accomplish throughout the day. Critically ill infants have complex physiological needs requiring advanced medical and nursing interventions to sustain life. Developing parent-infant relationships are influenced by the interactions within these early life experiences. ${ }^{1}$ Parents have identified communication and information provided by nurses as important factors influencing their experiences. ${ }^{2,3}$ Prior research establishes that caring behaviors directed at the infant's family are a significant component contributing to parent satisfaction with care. ${ }^{4,5}$ Additionally, the family unit has been identified to impact the health and well-being of the infant and conversely, the health of the infant has been shown to impact the health status of the family. ${ }^{6}$

\section{Aims}

The purpose of this study is to examine health care providers' beliefs and perceptions regarding providing family-centered care (FCC) in the NICU as well as variables that may influence provision of FCC.

\section{Methods}

This exploratory descriptive study used an online survey format. The survey comprised of 10 items from the Perceived Stress Scale, 10 items related to symptoms of burnout, a subscale of the Professional Quality of Life (ProQOL) scale and 27 items of the Family Nurse Caring Belief Scale (FNCBS). ${ }^{7}$ There were 6 items related to demographic characteristics, and 2 items regarding rationing care. Hospital IRB approval was obtained. An email which contained an information sheet describing the study with a link to a secure anonymous online survey through (www.qualtrics. com) was sent by an administrative assistant. No identifiable information was collected from participants. Consent was implied if respondents connected to the link to access the survey. Two reminder emails were sent after 1 and 3 weeks from the initial email invitation. The questionnaire was available to participants for a total of 4 weeks. SPSS version 25 was used for analysis of descriptive statistics, Pearson's correlation and one-way analysis of variance (ANOVA) were used to analyze participant responses.

\section{Results}

The sample consisted of 115 multidisciplinary participants working in a level IV NICU in southeastern United States. Participants report strong levels of beliefs of FCC and value its importance. The majority of participants (82\%) strongly agree that no matter how sick the infant is, he/she needs to be treated as an individual, the remaining $18 \%$ agreed with this statement. Participants strongly agreed (68\%) being available to families is an essential part of care in the NICU, the remaining $32 \%$ of participants agreed with this statement. A significant correlation (.001) exists between participant's stress composite score and FCC composite score indicating higher levels of stress are correlated with lower FCC scores. There were significant relationships between participant's years of experience (0.002) and education levels (0.005) in the NICU and FCC composite scores. There were also significant relationships between participant's years of experience (0.004) and FCC education (0.039) and Perceived Stress Scale composite scores. There were no relationships identified for the Professional Quality of Life (Burnout) Composite scores and demographic characteristics.

\section{Conclusion}

Health care professionals care greatly about providing FCC and understand its importance. Results suggest that participants agreed FCC is important yet feel inadequate staffing and inappropriate assignments may lead to rationing of care to infants and their families. Further research is needed given the limitations of this exploratory single-site study. It is essential to continue exploring factors that may lead to rationing of FCC, provision of FCC in the NICU is associated with reduced stress experiences, shorter durations of stay, and ultimately enhanced parent-infant relationships. ${ }^{8}$

References:

1. Vittner D, McGrath JM, Robinson J, Lawhon g, Cusson R, Eisenfeld L, Walsh S, Young $\mathrm{E}$, Cong X. Increases in oxytocin from skin-to-skin contact enhances development of parent-infant relationships. Biological Research for Nursing 2018, 20(1):54-62. http://doi: $10.1177 / 1099800417735633$

2. Lawhon g. Integrated nursing care: Vital issues important in the human care of the newborn. Seminars in Neonatology 2002,7:441-446.

3. Johnson AN. Promoting maternal confidence in the NICU. Journal of Pediatric Health Care 2008, 254-257.

4. Meiers SJ, Tomlinson P, Peden-McAlpine, C. Development of the Family Nurse Caring Belief Scale (FNCBS). Journal of Family Nursing 2007, 13(4): 484-502. http// doi: $10.1177 / 1074840707310734$

5. Yu X, Zhang J. Family centred care for hospitalized preterm infants: A systematic review and meta analysis. International Journal of Nursing Practice 2018, e12705.

6. Hinds PS, Feetham SL, Patterson Kelly K, Nolan MT. "The Family Factor" Knowledge Needed in Oncology Research. Cancer Nursing 2012, 35(1);1-2. http//doi:10.1097/ NCC.0b013e31823b561f

7. Magri EP. Psychometric Validation of the Family Nurse Caring Belief Scale in a Neonatal Nursing Population. Theses \& Dissertation 2014.

8. Staniszewska S, Brett J, Redshaw M, Psychol C, Hamilton K, Newburn, et al. The POPPY Study: Developing a Model of Family-Centered Care for Neonatal Units. Worldviews of Evidence-Based Nursing 2012, 243-255. http//doi:10.1111/j.1741-6787.2012.00253.x 